\section{Audit of care for epileptics in a general practice}

The care provided for epileptic patients has been criticised in the Reid report, ${ }^{1}$ by Hopkins and Scambler, ${ }^{2}$ and by Reynolds. ${ }^{3}$ Criticisms have included poor communication between specialists and general practitioners, lack of continuity in care, and inappropriate drug treatment. We report on an attempt to audit the nature and quality of the care provided for the known epileptics in a general practice of 8500 patients.

\section{Patients, methods, and results}

The known epileptics in the practice were identified from the practice morbidity register and their records reviewed. They were then divided into (1) patients who had received anticonvulsants or had suffered fits during the preceding two years, and (2) patients who had neither received anticonvulsants nor been known to have had fits during that time. All group 1 patients other than those under hospital surveillance were invited to attend for a clinical assessment, which included haematological investigations and measurement of serum anticonvulsant concentrations. Records of diagnosis and management were then reviewed at a meeting between the patient's general practitioner and a neurologist (PF).

Out of 64 patients recorded as epileptics 12 had died or left the practice at the time of the review. The remaining 52 gave a prevalence rate of $7 \cdot 6$

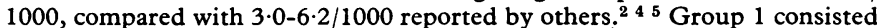
of 29 patients, of whom four were under regular hospital surveillance and four refused to attend for clinical assessment. The two groups had a similar age distribution. The table compares the place of diagnosis, nature of the fits, and the electroencephalographic record of the patients in the groups. Only

Epileptics in a general practice: comparison of patients in groups 1 and 2

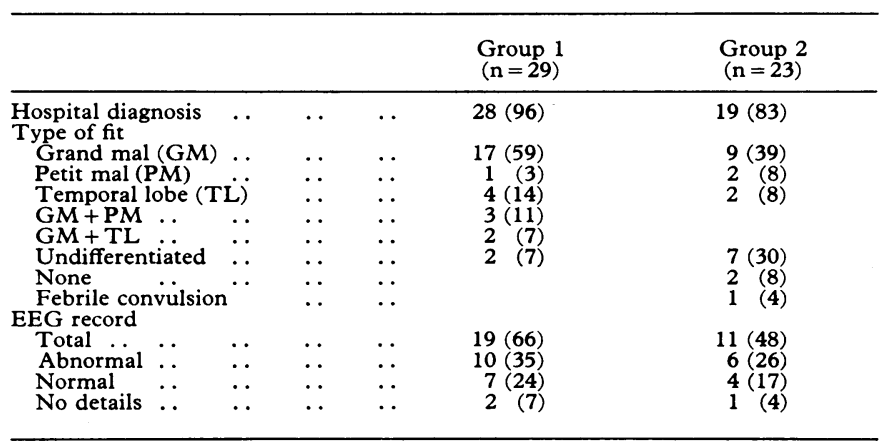

four of the 21 patients reviewed in group 1 had had a fit in the preceding year, and a further four had had fits within the preceding two years. Thirteen had had no fits during this period. Out of the four attending hospital the fits in three were very difficult to control, and the fourth, an 85-year-old man, had attended for 30 years for his medication and refused to discontinue this practice. The variety of drug regimens was wide. The only patients taking three or more drugs were those attending hospital. Nine patients had serum drug concentrations within the therapeutic range, while in the remaining 12 at least one of the drugs prescribed produced subtherapeutic concentrations. No patient had toxic concentrations. Three patients taking phenobarbitone complained of drowsiness. In three others behaviour problems may have been exacerbated by the drug treatment. Haemoglobin and plasma folate, calcium, and phosphate concentrations were normal in all patients. As a result of the review two patients were investigated further and one was found to have a meningioma. Withdrawal of treatment was recommended for four patients and an alteration in treatment suggested for five.

\section{Comment}

This review does not support the rather dismal reports about the quality of control of epilepsy. Thus most patients were free from fits (only seven out of 52 had had a fit in the preceding year), only six had evidence of possible side effects of drugs, and none had any haematological abnormalities. After the combined clinical review one patient was found to have a meningioma and nine had their medication modified. The difficulty in controlling the fits in three of the four patients attending hospital compared with the 48 patients cared for in general practice shows the danger of generalising from hospital experience of epilepsy. An important lesson underlined by this audit was the good prognosis associated with one or two epileptic manifestations in early life even when the original diagnosis was supported by electroencephalographic abnormalities.

We thank our partners, Drs W S Marson, C J Watkins, and H G Gage for their co-operation, and Dr Felicity Reynolds for measuring serum anticonvulstant concentrations. The cost of the study was in part defrayed by a grant from the Department of Health and Social Security.

${ }^{1}$ Advisory Committee on the Health and Welfare of Handicapped Persons, Central Health Services Council, People with Epilepsy. London, HMSO, 1969.

${ }^{2}$ Hopkins, A, and Scambler, S, Lancet, 1977, 1, 183.

3 Reynolds, E H, Lancet, 1978, 11, 721.

4 Morbidity Statistics for General Practice, Second National Study, 1970-71. London, HMSO, 1974.

5 Pond, D A, Bidwell, B H, and Stein, L, Psychiatria, Neurologia, Neurochirurgia, 1960, 63, 217 .

(Accepted 7 August 1979)

General Practice Teaching and Research Unit, St Thomas's Hospital Medical School, London SE11 4TH

L I ZANDER, MB, MRCGP, senior lecturer

H GRAHAM, MB, DPH, general practitioner

D C MORRELL, FRCP, FRCGP, Wolfson professor

P FENWICK, MB, MRCPSYCH, consultant neurologist

\section{Extrapyramidal syndrome with sodium valproate}

The anatomical interrelationship between dopaminergic and gammaaminobutyric acid (GABA) neurons and evidence for functional antagonism between these systems have led investigators to suggest that conditions thought to be related to dopaminergic hyperfunction such as schizophrenia and tardive dyskinesia might be beneficially affected by GABA facilitation. ${ }^{1}$ Trials of putative GABA agonists such as baclofen ${ }^{2}{ }^{3}$ and sodium valproate, ${ }^{4}$ and of the specific GABA agonist muscimol ${ }^{5}$ represent attempts to use this approach in these conditions. The results of such studies have, however, often been contradictory.

We report here an extrapyramidal syndrome emerging during a trial in which the effect of sodium valproate on schizophrenic psychopathology was being assessed.

\section{Case report}

A 52-year-old man with a history of chronic schizophrenia from early adult life was treated with sodium valproate alone. He had been admitted to hospital several times before, when standard neuroleptic agents had diminished but never completely abolished his symptoms. By the fourth day of treatment with sodium valproate $1 \mathrm{~g}$ /day the patient developed a pronounced bilateral, low amplitude Parkinsonian-like tremor of the arms. Associated with this tremor was a circumoral tremor of the same frequency. Cogwheel rigidity of the arms was demonstrable only after reinforcement. The intensity of these signs increased with increasing dosage. At $1.5 \mathrm{~g} /$ day he was given $2 \mathrm{mg}$ benztropine intravenously with no clinical effect. At $2 \mathrm{~g} /$ day of valproate trihexyphenidyl $2 \mathrm{mg}$ by mouth three times daily was added to his regimen, also without clinical effect. Valproate was discontinued gradually over three days. The tremor cleared two days after the last dose. Valproate had no therapeutic effect on the patient's schizophrenia.

After a five-day drug free period in which no change in his condition occurred the patient began a therapeutic trial of the dopamine antagonist metoclopramide. After six days at a dose of $90 \mathrm{mg}$ by mouth four times daily an identical syndrome emerged. At this time trihexyphenidyl $2 \mathrm{mg}$ by mouth three times daily completely cleared the patient's drug-induced resting tremor, which re-emerged in a milder form when the dose of metoclopramide reached $1 \mathrm{~g} /$ day. Metoclopramide treatment produced partial improvement 